\title{
COMPLETITUD DEL CATÁLOGO DE LA RED SISMOLÓGICA NACIONAL DE COSTA RICA DURANTE 1975-2014
}

\author{
Mario Arroyo $^{1 *}$; Kevin Godínez ${ }^{1}$; Lepolt Linkimer ${ }^{1}$ \\ DOI: http://dx.doi.org/10.18273/revbol.v39n3-2017006 @ (1)
}

Forma de citar: Arroyo, M., Godínez, K., y Linkimer, L. 2017. Completitud del catálogo de la Red Sismológica Nacional de Costa Rica durante 1975-2014. Boletín de Geología, 39(3): 87-98.

\section{RESUMEN}

En este artículo se evalúa la estabilidad de la tasa media de ocurrencia de sismos y la distribución temporal de la sismicidad en Costa Rica a partir del método de Stepp, con el fin de determinar la magnitud de completitud del catálogo sísmico de la Red Sismológica Nacional (RSN) y la relación Gutenberg-Richter para el periodo 19752014. El catalogo fue depurado de acuerdo con varios criterios y homogeneizado a magnitud momento $(M w)$. La magnitud de completitud del catálogo total obtenida es de 5,0, pero puede disminuir hasta 3,0 para ciertos periodos de tiempo. Los resultados reflejan la mejora en la detección de sismos debido al cambio de sistemas analógicos a digitales a partir de 1990 y el aumento considerable en el número de estaciones a partir del año 1995. El valor $b$ obtenido para todo el catálogo es 0,96 , el cual es muy similar a valores regionales obtenidos en estudios previos, no obstante deben considerarse las limitaciones discutidas en el artículo para su correcta interpretación.

Palabras clave: magnitud de completitud; sismicidad en Costa Rica; método de Stepp.

\section{COMPLETENESS OF THE COSTA RICA NATIONAL SEISMOLOGICAL NETWORK CATALOG DURING 1975-2014}

\begin{abstract}
This study evaluates the stability of the mean earthquake occurrence rate and the temporal distribution of seismicity in Costa Rica from the Stepp method, in order to determine the completeness magnitude of the seismic catalog of the National Seismological Network (RSN) and the Gutenberg-Richter relationship for the period 1975-2014. The catalog was filtered using several criteria and homogenized to moment magnitude (Mw). The magnitude of completeness obtained for the entire catalog is 5.0, but it could be as low as 3.0 for certain periods of time. These results show the improvement in seismic detection due to the change from analogue to digital systems since 1990 and the considerable increase in the number of stations since 1995 . The value $b$ obtained for this catalog is 0.96 , which is very similar to regional $b$ values obtained in previous studies, nevertheless, the limitations discussed in this paper have to be considered in order to interpret this value.
\end{abstract}

Keywords: completeness magnitude; seismicity of Costa Rica; Stepp method.

\footnotetext{
${ }^{1}$ Sección de Sismología, Vulcanología y Exploración Geofísica, Escuela Centroamericana de Geología y Red Sismológica Nacional, Universidad de Costa Rica. San Pedro, Costa Rica. (*)mario.arroyosolorzano@ucr.ac.cr, kevin.godinezrodriguez@ucr.ac.cr, lepolt.linkimer@ucr.ac.cr
} 


\section{INTRODUCCIÓN}

La determinación de la Magnitud de Completitud $(M C)$ de un catálogo sísmico es esencial para analizar la sismicidad de una región. $M C$ se define como la magnitud más baja en la cual el $100 \%$ de los sismos son detectados exitosamente dentro de una región y periodo de tiempo (Woessner y Wiemer, 2005). $M C$ varía en el tiempo y el espacio y depende de muchos factores que afectan la capacidad de detección de una red sismológica, como son: la densidad y distribución de estaciones sísmicas, el tipo de instrumentación usada, la eficiencia del envío de datos desde las estaciones al centro de procesamiento, las prácticas y procedimientos de detección de sismos, entre otros (Chouliaras, 2009; Mignan y Woessner, 2012; Wiemer y Wyss, 2000).
Costa Rica se caracteriza por una alta sismicidad debido a su localización en una zona de subducción y cerca del punto triple de las placas del Coco, Caribe y Nazca (FIGURA 1). La sismicidad es especialmente abundante a lo largo de la Fosa Mesoamericana y en la zona sismogénica interplacas, donde se subduce la placa del Coco debajo de la placa Caribe y la microplaca de Panamá. Además, la sismicidad es alta a lo largo de la Zona de Fractura de Panamá (ZFP), la cual, sirve de límite entre las placas del Coco y Nazca. Asimismo, los simos son frecuentes a lo largo del Cinturón Deformado del Norte de Panamá (CDNP) y el Cinturón Deformado del Centro de Costa Rica (CDCCR), los cuales son anchas zonas de deformación cortical con una alta densidad de fallas activas (Fan et al., 1993; Goes et al., 1993; Marshall et al., 2000; Montero, 2001) (FIGURA 1).



FIGURA 1. Mapa tectónico simplificado de Costa Rica y ubicación de las estaciones de la RSN durante el año 2014. La región contenida dentro de la línea gruesa punteada representa el Cinturón Deformado del Centro de Costa Rica (CDCCR) de acuerdo con Montero y Rojas (2014). La línea discontinua representa el límite noreste simplificado del Bloque Antearco Centroamericano a lo largo de las Fallas del Arco Volcánico (FAV) de acuerdo con Franco et al. (2012). Otras estructuras tectónicas son: Cinturón Deformado del Norte de Panamá (CDNP), Cadena volcánica submarina de Fisher (F), Plateau de Quepos (PQ) y Zona de Fractura de Panamá (ZFP).

La Red Sismológica Nacional (RSN: UCR-ICE) es uno de los entes dedicados a la vigilancia sísmica de Costa Rica. Fue creada mediante un convenio entre la Sección de Sismología, Vulcanología y Exploración
Geofísica (SSVEG) de la Escuela Centroamericana de Geología de la Universidad de Costa Rica (UCR) y el Área de Amenazas y Auscultación Sísmica y Volcánica (AAASV) del Instituto Costarricense de Electricidad 
(ICE). La SSVEG y el AAASV funcionan desde 1973 y 1974, respectivamente, y el centro de registro conjunto de la RSN, con sede en la UCR, opera desde 1982.

El catálogo sísmico existente de la RSN incluye los sismos ocurridos a partir del año 1974. La cantidad y distribución de las estaciones con las que se han localizado los sismos de este catálogo ha variado significativamente a lo largo de la historia de la RSN. En 1976, la red de estaciones contaba únicamente con cinco instrumentos en la parte central de Costa Rica (Morales, 1986), número que creció paulatinamente hasta alrededor de 60 estaciones, ubicadas en todo el país para el 2014. Esta distribución heterogénea de las estaciones desde un punto de vista geográfico y temporal es una situación común en las redes sismológicas e implica que muchos sismos no son detectados en ciertos rangos de tiempo y, en ciertas regiones geográficas, especialmente los eventos de magnitud baja que ocurren durante los periodos de tiempo en los cuales las redes operan con pocos instrumentos.

Partiendo de lo anterior, se analiza la sismicidad localizada por la RSN en Costa Rica con el fin de determinar la $M C$ para el periodo de tiempo 19752014. Para el cálculo de la $M C$ en este estudio se ha elegido utilizar el tradicional método de Stepp (1972) con el fin de ofrecer un primer resultado para todo el catálogo de la RSN que sirva de marco de referencia para futuros cálculos con otras metodologías, como con el programa Zmap de Wiemer (2001). Adicionalmente, se calcula la relación Gutenberg-Richter con ciertas limitaciones que se discuten en el artículo. Se deja por fuera del alcance de esta investigación la zonificación por fuentes sísmicas o geográficas, ya que el objetivo en esta ocasión es documentar los valores generales para todo el catálogo. La determinación de la $M C$ y los parámetros de la relación Gutenberg-Richter son de suma importancia para la práctica sismológica, pues son la base de los estudios de amenaza sísmica de una región particular.

\section{EL CATÁLOGO DE SISMOS DE LA RSN}

El catálogo sísmico de la RSN analizado en este estudio está compuesto por 111.850 sismos que ocurrieron entre el 4 de noviembre de 1974 y el 31 de diciembre del 2014. Este catálogo incluye las características básicas de los sismos localizados, esto es, la fecha, hora, coordenadas del epicentro (latitud y longitud), profundidad del hipocentro y en la mayoría de los casos su magnitud.

Dado que el catálogo de la RSN contiene sismos regionales (a nivel Centroamericano) y telesismos (eventos a nivel global), se restringió el área de estudio para enfocar el análisis de la sismicidad dentro del territorio de Costa Rica. De esta forma, se analizaron únicamente los sismos ubicados entre las coordenadas: $7,0^{\circ}$ y $11,5^{\circ}$ de latitud $\mathrm{N}$ y $86,5^{\circ}$ y $82,0^{\circ}$ de longitud W. Esta porción del catálogo está compuesto por 68.022 sismos. Asimismo, se analizó la sismicidad para años completos, es decir, comenzando el 1 de enero de 1975 y hasta el 31 de diciembre del 2014.

Además de la restricción temporal y geográfica, se tomaron en cuenta otros criterios para descartar los sismos con localizaciones de baja calidad. De esta forma, se obviaron del análisis los eventos localizados con menos de cuatro estaciones y también, los caracterizados por un valor de la raíz cuadrática media (RMS) mayor a 1,0. Tampoco fueron tomados en cuenta aquellos que no contaban con una estimación de magnitud. Adicionalmente, se realizó una inspección de la profundidad, y fueron descartados aquellos sismos con profundidades atípicas, considerando el espesor de la corteza y profundidad de la losa de la placa del Coco que se subduce bajo Costa Rica (Lucke, 2014). El catálogo filtrado de acuerdo con las restricciones y los criterios mencionados, está compuesto por 62.514 sismos (TABLA 1).

TABLA 1. Cantidad de sismos en rangos de cinco años del catálogo depurado de la RSN.

\begin{tabular}{cc}
\hline Intervalo de tiempo & $\begin{array}{c}\text { Cantidad total de sismos } \\
\text { registrados }\end{array}$ \\
\hline $2010-2014$ & 9.689 \\
$2005-2009$ & 13.664 \\
$2000-2004$ & 16.705 \\
$1995-1999$ & 10.709 \\
$1990-1994$ & 8.389 \\
$1985-1989$ & 2.465 \\
$1980-1984$ & 733 \\
$1975-1979$ & 160 \\
Total & 62.514 \\
\hline
\end{tabular}

Otro aspecto tratado en la preparación del catálogo fue la uniformización de la magnitud, ya que la determinación de la misma ha variado a lo largo de la historia de la RSN. Antes del 2012, se determinaba principalmente la magnitud coda $(M d)$ o la magnitud local $(M L)$. Sin embargo, desde el 2012, se estima únicamente la magnitud momento $(M w)$. Con el fin de realizar un análisis homogeneizado de la magnitud del catálogo, se procedió a convertir las magnitudes $M L$ y $M d$ a $M w$. 
Para la conversión de las magnitudes, se usaron las relaciones determinadas por Rojas (1993) y Rojas et al. (1993).

\section{RESULTADOS: COMPLETITUD DEL CATÁlOGO}

Para la determinación de la $M C$ se utilizó la metodología de Stepp (1972). Este método evalúa la estabilidad de la tasa media de la ocurrencia de sismos en un rango de magnitud y en una serie de periodos de tiempo predefinidos. Stepp (1972) supone que la tasa de ocurrencia de los sismos en una región es uniforme o se mantiene constante a través del tiempo. Además, infiere que la ocurrencia de terremotos sigue una distribución de Poisson, es decir, que hace referencia a variables sometidas a influencias, impactos o fenómenos aleatorios que evolucionan en función de una variable, que puede ser el tiempo o el espacio (Cárdenas et al., 2010).

De acuerdo con Stepp (1972), la desviación estándar $(s)$ de la tasa media de ocurrencia de sismos $(M)$ durante un periodo de tiempo acumulado $(T)$ estaría dada por:

$$
s=\sqrt{\frac{M}{T}}
$$

Si se asume que la tasa media de ocurrencia de sismos es constante, entonces la desviación estándar varía como $1 / \sqrt{ } \mathrm{T}$. Por consiguiente, un rango de magnitud durante un periodo de tiempo es considerado en completitud cuando la tendencia de la desviación estándar, es igual o muy similar a la de $1 / \sqrt{ } \mathrm{T}$ (Nasir et al., 2013). Si esta tendencia no se cumple, indicaría que el intervalo de tiempo no es suficiente o que el rango de magnitud bajo estudio no es completo (Caneva et al., 2003).

El análisis del catálogo de la RSN se realizó para intervalos de tiempo de cinco años desde 1975 al 2014, es decir, se consideraron los siguientes ocho intervalos de tiempo: 1975-1979, 1980-1984, 1985-1989, 19901994, 1995-1999, 2000-2004, 2005-2009 y 2010-2014. Además, se trabajó con 12 rangos de magnitud, que incluyen un rango de $M w \leq 1,9$, diez rangos desde 2,0 a 6,9 subdivididos en intervalos de 0,5 (i.e., 2-2.4, 2.5$2.9, \ldots 6,5-6,9)$ y un rango para $M w \geq 7,0$.

Con el fin de seguir la metodología de Stepp (1972), se crearon tablas de análisis para la determinación del número de eventos acumulado, la tasa media de ocurrencia y la desviación estándar, como se ejemplifica para el intervalo de $M w$ de 5,0 a 5,4 en la TABLA 2. A partir de los datos estadísticos obtenidos, se realizó una comparación gráfica entre $s$ y $1 / \sqrt{ } \mathrm{T}$ para todos los rangos temporales y de $M w$ predefinidos.

TABLA 2. Ejemplo de los resultados obtenidos con el método de Stepp (1972) para el intervalo de $M w$ 5,0 - 5,4.

\begin{tabular}{cccccc}
\hline Intervalo de tiempo & Tiempo acumulado (T) & $\mathbf{1 /} \mathbf{T}$ & $\begin{array}{c}\text { Número de eventos } \\
\text { acumulado (N) }\end{array}$ & $\begin{array}{c}\text { Tasa media de } \\
\text { ocurrencia (M) }\end{array}$ & $\begin{array}{c}\text { Desviación } \\
\text { estándar (s) }\end{array}$ \\
\hline $2010-2014$ & 5 & 0,45 & 56 & 11,20 & 1,50 \\
$2005-2009$ & 10 & 0,32 & 122 & 12,20 & 1,10 \\
$2000-2004$ & 15 & 0,26 & 189 & 12,60 & 0,92 \\
$1995-1999$ & 20 & 0,22 & 237 & 11,85 & 0,77 \\
$1990-1994$ & 25 & 0,20 & 332 & 13,28 & 0,73 \\
$1985-1989$ & 30 & 0,18 & 369 & 12,30 & 0,64 \\
$1980-1984$ & 35 & 0,17 & 414 & 11,83 & 0,58 \\
$1975-1979$ & 40 & 0,16 & 423 & 10,58 & 0,51 \\
\hline
\end{tabular}

Para inspeccionar la relación entre la desviación estándar y $1 / \sqrt{ } \mathrm{T}$, se graficó el $\log 10$ de las desviaciones estándar por periodos de tiempo para cada rango de $M w$, así como el valor de $1 / \sqrt{ }$ T que sirve de referencia (FIGURA 2). Dado que visualmente el patrón de las curvas es muy uniforme, se procedió a cuantificar la tendencia entre la curva de la desviación estándar en comparación con la de $1 / \sqrt{ } \mathrm{T}$, a través de la determinación de un ajuste potencial para cada una de las curvas graficadas. Se tomó como criterio, un alejamiento aceptable de 0,01 con relación al $R^{2}$ de $1 / \sqrt{ } T$, pues se consideró que este valor aún representa la tendencia que sigue $1 / \sqrt{ } \mathrm{T}$. Es así como los rangos de $M w$ con un $\mathrm{R}^{2} \geq 0,99$ se consideraron como en completitud para el periodo de tiempo analizado.

En la FIGURA 2A, se aprecian los datos graficados desde 1975 hasta el 2014. En esta figura se muestran, además, los $\mathrm{R}^{2}$ para este rango de tiempo. Se observa como los rangos $5,0 \leq M w \leq 5,4,5,5 \leq M w \leq 5,9$ y 6,0 $\leq M w \leq 6,4$ están en completitud, ya que sus $\mathrm{R}^{2}$ son de 0,99 para todo el periodo 1975-2014. Es importante aclarar que para los sismos de $M w \geq 6,5$ el ajuste 
potencial de la desviación estándar no es el óptimo, ya que los sismos de alta $M w$ son poco frecuentes dentro de la ventana de tiempo analizada. Tomando en cuenta la pequeña muestra de sismos de $M w \geq 6,5$ disponibles (sólo 13), se requiere de un periodo de tiempo mucho mayor para poder obtener una relación que siga la misma tendencia de $1 / \sqrt{ } \mathrm{T}$. No obstante, se considera que el catálogo de la RSN si está en completitud para $M w \geq 6,5$, dado que la metodología de Stepp (1972) así lo determina para magnitudes menores en el rango de $M w$ desde 5,0 a 6,4. El caso particular de $M w$ 5,0-5,9 en el periodo 1975-1979, no se considera con certeza en completitud, debido a observaciones adicionales que se presentan más adelante en esta misma sección (TABLA 3).

Una vez determinada la $M C$ de 5,0 para el periodo 19752014 (FIGURA 2A), se procedió a inspeccionar con más detalle periodos de tiempo menores, en los que se determinó el ajuste potencial de la curva de $s$ y su relación con $1 / \sqrt{ } \mathrm{T}$, buscando los rangos de tiempo para los cuales el ajuste de $\mathrm{R}^{2}$ es $\geq 0,99$ (FIGURA 2B). Se tomó como tiempo mínimo aceptable un periodo de al menos 20 años para el análisis de sismos de $M w \leq 6,4$. Es así como se determinó que para el rango 4,5 $\leq M w \leq 4,9$ el catálogo es completo para el periodo 1980-2014, para los rangos $4,0 \leq M w \leq 4,4$ y $3,5 \leq M w \leq 3,9$ el catálogo es completo para el periodo 1990-2014 y para el rango 3,0 $\leq M w \leq 3,4$ el catálogo es completo para el periodo 1995-2014. Para los sismos con $M w \leq 2,9$ se estableció que el catálogo no se encuentra en completitud (TABLA 3). En particular, el rango de $M w 2,5-2,9$ posee un $\mathrm{R}^{2}$ es $\geq 0,99$ para el periodo 2000-2014, no obstante, este rango de Mw no se considera con certeza en completitud, ya que no cumple con el requisito de tiempo mínimo aceptable de 20 años para un $R^{2} \geq 0,99$.

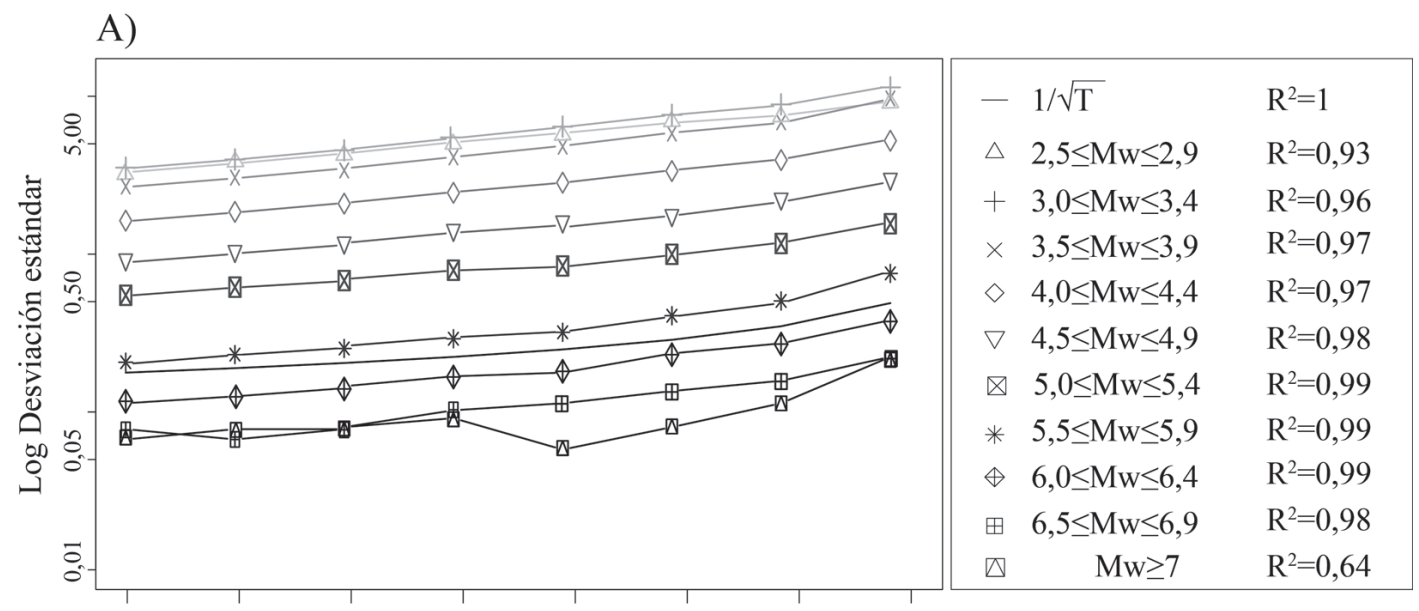

1975-1979 1980-1984 1985-1989 $\quad 1990-1994 \quad 1995-1999 \quad 2000-2004 \quad 2005-2009 \quad 2010-2014$

B)



FIGURA 2. Resultados del método de Stepp (1972). A. Desviación estándar de la tasa de frecuencia de sismos para rangos desde Mw 2,5 para todo el periodo de estudio con su respectivo $\mathrm{R}^{2}$ y $1 / \sqrt{ } \mathrm{T}$. B. Desviación estándar de la tasa de frecuencia de sismos para rangos de $M w$ considerados en completitud $\left(\mathrm{R}^{2}=0,99\right)$ con su respectivo $1 / \sqrt{\mathrm{T}}$ para un tiempo mínimo de 20 años. 
TABLA 3. Propuesta de completitud del catálogo para diferentes rangos de $M w$ e intervalos de tiempo. Ver FIGURA 5.

\begin{tabular}{cc}
\hline Rango (Mw) & Intervalo de tiempo \\
\hline$<2,5$ & Incompleto \\
$2,5-2,9$ & $i 2000-2014 ?$ \\
$3,0-3,4$ & $1995-2014$ \\
$3,5-3,9$ & $1990-2014$ \\
$4,0-4,4$ & $1990-2014$ \\
$4,5-4,9$ & $1980-2014$ \\
$5,0-5,9$ & ¿1975-1979? y $1980-2014$ \\
$\geq 6,0$ & $1975-2014$ \\
\hline
\end{tabular}

Para respaldar los resultados arrojados por el método de Stepp (1972), se inspeccionó la constancia de la cantidad de sismos a través del tiempo, según rangos de $M w$ a partir de 4,0 (FIGURAS 3 y 4). Para cada gráfico se determinó la pendiente $(m)$, de forma que si $m \leq 0,1$, la tasa de sismicidad es considerada como constante durante el periodo analizado. Lo obtenido en esta inspección apoya el resultado de la metodología de Stepp (1972) para una $M C$ de 5,0, ya que arriba de esta magnitud, $m$ es casi cero, lo que implica muy poca variación de la tasa de sismicidad. Por el contrario,

A) $4,0<\mathrm{Mw}<4,9$



C) $6,0<\mathrm{Mw}<6,9$

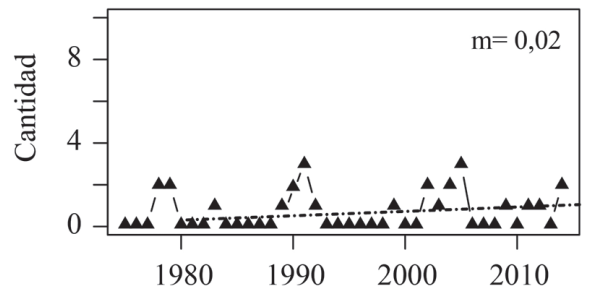

debajo de la $M C$ de 5,0 la $m$ es alta, mostrando poca constancia en la tasa de sismicidad y sugiriendo un catálogo incompleto. Por ejemplo, para el rango de $M w$ 4,0-4,9 la $m$ es muy alta $(m=6)$, es decir, se observa un aumento considerable en la detección de sismos a través del tiempo, especialmente después de 1990 (FIGURA 3A).

Una observación importante que resulta de la inspección de la constancia de sismos en el tiempo, es que para el rango de $M w$ 5,0-5,9 durante el periodo de 1975-1979 existe una muestra muy baja de sismos (FIGURA 3B). Según el método de Stepp (1972) este rango de tiempo y magnitud es considerado en completitud (FIGURA 2), sin embargo, este resultado se encuentra fuertemente influenciado por la muestra completa de sismos proveniente del periodo 1980-2014. Debido a la poca cantidad de sismos de $M w$ 5,0-5,9 durante el periodo 1975-1979, se determinó la m excluyendo este periodo. Los resultados obtenidos muestran que, obviando ese periodo de tiempo, la cantidad de sismos es casi constante ( $m=0,09$, FIGURA 3B). Con base en estas observaciones, se considera que el periodo de completitud para el rango de $M w 5,0-5,9$ es de 1980 2014 (TABLA 3).



D) $\mathrm{Mw}>7,0$



FIGURA 3. Cantidad de sismos según $M w$ en el periodo 1975-2014. A. $M w$ de 4,0 a 4,9. B. $M w$ de 5,0 a 5,9. C. $M w$ de 6,0 a 6,9. D. $M w \geq 7,0$. La línea discontinua denota el ajuste lineal de la cantidad de sismos en el tiempo a partir de 1980 y $m$ representa la pendiente de dicho ajuste lineal en cada caso.

Adicionalmente, se inspeccionó con más detalle la muestra de sismos con $M w$ inmediatamente inferior a la $M C$ de 5,0 (FIGURA 4). La intención de este análisis fue revisar la constancia en el tiempo de cada $M w$ en variaciones de 0,1 , en caso de que el valor de la $M C$ fuese inferior al arrojado por la metodología de Stepp (1972), la cual se basa en rangos mayores de $M w$ de
0,5 . Se calculó la m para cada gráfico de $M w$ desde 4,7 a 5,0, y se consideró la sismicidad como constante si $m$ $<0,1$ durante el periodo analizado (de 1980 al 2014). Los resultados obtenidos muestran que la detección de sismos es prácticamente constante para la $M w$ de 5,0 y que $m$ aumenta progresivamente conforme disminuye $M w$ ( $m$ es de 0,10, 0,11 y 0,21 para un $M w$ de 4,9, 4,8 
y 4,7, respectivamente). Estos resultados apoyan los obtenidos por el método de Stepp (1972) y sugieren

A) $\mathrm{Mw} 4,7$



C) $\mathrm{Mw} 4,9$



que los sismos de $M w$ de 4,8 y 4,9 están cerca de la completitud.



D) $\mathrm{Mw} 5,0$



FIGURA 4. Cantidad de sismos de $M w 4,7$ a 5,0 en el periodo 1975-2014. A. $M w$ 4,7. B. $M w$ 4,8. C. $M w$ 4,9. D. $M w$ 5,0. La línea discontinua denota el ajuste lineal de la cantidad de sismos en el tiempo a partir de 1980 y m representa la pendiente del ajuste lineal en cada caso.

Con base en lo anterior, se concluye que la $M C$ de 5,0 es un valor robusto para el periodo 1980-2014. Un resumen de la completitud del catálogo sísmico de la RSN se muestra en la TABLA 3 y FIGURA 5. El cambio de sistemas de analógicos a digitales, a partir de 1990, y el aumento considerable en el número de estaciones a partir de 1995 están reflejados en los resultados obtenidos que muestran un valor menor de la $M C$ a partir de los años señalados (TABLA 3 y FIGURA 5).



FIGURA 5. Resumen de completitud del catálogo de sismos de la RSN.
La distribución geográfica de la porción del catálogo con $M C>5,0$ se muestra en la FIGURA 6 subdividida en cuatro décadas desde 1975 hasta el 2014. Se observa que la sismicidad de mayor magnitud ha ocurrido principalmente a lo largo de la costa Pacífica, asociada con la subducción de la placa del Coco bajo la placa Caribe y la microplaca de Panamá. En el catálogo analizado, los terremotos de mayor magnitud han sido: Sámara de 1978 (Mw 6,8, FIGURA 6A), Golfito de 1983 ( $M w 7,4$, FIGURA 6A), Cóbano de 1990 (Mw 7,3, FIGURA 6B), Limón de 1991 (Mw 7,7, FIGURA 6B, Goes et al., 1993), Quepos de 1999 (Mw 6,9, FIGURA 6C) y Sámara del 2012 (Mw 7,6, FIGURA 6D).

\section{RELACIÓN GUTENBERG-RICHTER}

La relación Gutenberg y Richter (1944) muestra la distribución relativa del tamaño de los sismos. Esta relación está definida por:

$$
\log (N)=a-b \cdot M C
$$

Donde los parámetros $a$ y $b$ corresponden con la ordenada en el origen y la pendiente, respectivamente. De acuerdo con esta relación, los sismos grandes son poco frecuentes en comparación con los pequeños y conservan una estricta relación proporcional entre ellos. 
A) $1975-1984$



C) $1995-2005$



Magnitud (Mw)



B) $1985-1994$

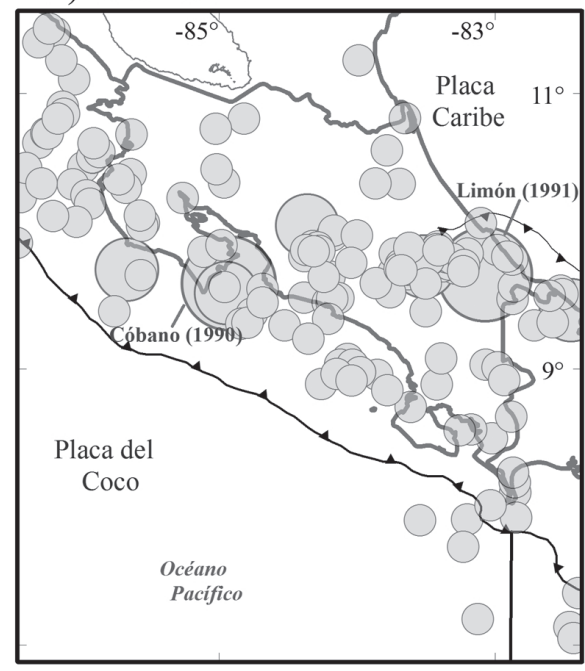

D) $2005-2014$


FIGURA 6. Distribución geográfica de la sismicidad por décadas durante 1975-2014 de los sismos con MC > 5. A. $1975-1984$. B. 1985-1994. C. 1995-2004. D. 2005-2014.

La relación de Gutenberg-Richter calculada usando el catálogo de la RSN durante 1975-2014 se muestra en la FIGURA 7. De la curva de frecuencia no acumulada, se observa que la mayoría de los sismos del catálogo poseen una $M w$ entre 2,9 y 3,2. Usando la $M C$ de 5,0, determinada con el método Stepp (1972), se realizó un ajuste lineal con mínimos cuadrados para la determinación de los parámetros de $a$ y $b$ (FIGURA 7). Estos parámetros obtenidos corresponden con valores regionales "promedio", ya que toman en cuenta los sismos de todas las profundidades y fuentes sísmicas en la región de estudio. Es importante mencionar que la relación Gutenberg-Richter se realizó a partir del catálogo completo, considerando la $M C$ obtenida a partir de Stepp (1972), sin eliminar los premonitores y las réplicas de los terremotos, los cuales pueden afectar los parámetros $a \mathrm{y}$ $b$ (Knopoff, 2000). Por esta razón, estos valores sólo se presentan con fines ilustrativos para mostrar la relación Gutenberg-Richter regional del catálogo y se recomienda evitar su interpretación tectónica (FIGURA 7). 




FIGURA 7. Relación de Gutenberg-Richter para el catálogo de la RSN en el periodo 1975-2014. Se muestra con fines ilustrativos el ajuste lineal por mínimos cuadrados de la cantidad de sismos acumulada para una $M C$ de 5,0. El valor $b$ obtenido por el método de Aki (1965) es 0,96.

El cálculo del valor de $b$ para la región por medio de una regresión de mínimos cuadrados contradice la suposición de que los datos son independientes (Naylor et al., 2010). Debido a ello, este valor se recalculó a través de la metodología de máxima verosimilitud, propuesta por Aki (1965), en la cual se asume que los datos son distribuidos exponencialmente y que la magnitud máxima es infinita (i.e., lo más probable es que la muestra sobre la que se calcula el valor de $b$ no es suficientemente grande para probar la máxima magnitud). La estimación por este método, consiste en buscar el valor de $b$ que maximice la función de probabilidad, por medio de la fórmula:

$$
b=\frac{\log e}{M m-(M C-\Delta m / 2)}
$$

Donde $\mathrm{Mm}$ es la magnitud media del grupo de sismos a partir de la $M C$ y $\Delta m$ es el ancho del intervalo de magnitud analizado (Naylor et al., 2010). Tomando en cuenta un $M m$ de 5,40 para el catálogo en completitud y una $M C$ de 5,0 , el valor de $b$ obtenido por este último método es de 0,96. Este valor se encuentra dentro del rango de valores publicados para la región de Costa Rica que varían entre 0,48 y 1,40 para áreas y fuentes sísmicas específicas (Rojas et al., 1993; Laporte et al., 1994; Benito et al., 2012). Además, el resultado obtenido es similar al valor de $b$ promedio para diferentes subregiones dentro de Costa Rica presentado en estudios previos, por ejemplo: 0,97 en Rojas et al. (1993), 0,91 en Laporte et al. (1994) y 0,93 en Benito et al. (2012), no obstante deben de considerarse las limitaciones que se explican en la siguiente sección para su correcta interpretación.

\section{DISCUSIÓN SOBRE EL SESGO DEL VALOR $b$}

El valor $b$ es la pendiente de la relación GutenbergRichter (ecuación 2) y por consiguiente, este valor describe la abundancia relativa de eventos grandes en comparación con los pequeños para el catálogo estudiado. Al caracterizar la proporción de las magnitudes de los sismos, el valor $b$ en cierta forma representa el estado de esfuerzos al que está sometido una región particular (Benito y Jiménez, 1999; Kulhanek, 2005; Sobiesiak, 2005) y por esto además está influenciado por la extensión de la ventana de tiempo en que es analizado (i.e., por el momento en el ciclo sísmico en que es observado). Además, el valor $b$ depende secundariamente de la heterogeneidad del material, por lo que aumenta con la heterogeneidad creciente (Sobiesiak, 2005).

Si una red sismológica tuviera la capacidad de detectar absolutamente todos los sismos, el valor de $b$ sería un fiel reflejo de la realidad tectónica de la región para la ventana de tiempo de observación. Para poder realizar un estudio que contemple toda la gama de magnitudes, se debería establecer un lugar y momento de tiempo en los cuales se tuviera certeza de que se ha mantenido una capacidad de registro de sismicidad instrumental constante en dicho lapso (Benito et al., 2012; Mendoza, 2012; Valladares, 2013). Desafortunadamente, aún es difícil que una red sismológica alcance la detección total de los sismos para periodos considerables de tiempo. Por esta razón, el concepto de la $M C$ intenta solventar este sesgo inherente en el cálculo de $b$ para los catálogos sísmicos. Así, cada estudio aproxima el valor 
de $b$ para el catálogo con base en la elección de una magnitud mínima de detección confiable en el periodo de tiempo analizado.

Existen varios factores limitantes que afectan el cálculo de $b$ presentado en este estudio. Como se mencionó en la sección anterior, la relación Gutenberg-Richter se construyó a partir del catálogo completo sin eliminar premonitores y réplicas de los terremotos, lo cual para algunos autores viola la "independencia" de cada evento sísmico si estos siguieran una distribución de Poisson, y por ende, esto afectaría el cálculo de la proporcionalidad de la magnitud entre los sismos (Knopoff, 2000). No obstante, algunos estudios realizan un cálculo del valor de $b$ usando los terremotos y sus réplicas (Sobiesiak, 2000).

El cálculo de la magnitud de los sismos es otro factor limitante si este no ha sido uniforme a lo largo de la historia de la red. En nuestro estudio, este aspecto se intenta resolver al convertir las magnitudes a un mismo tipo $M w$, como se mencionó en la segunda sección de este artículo. Otra restricción es el tiempo de observación de solamente 40 años, el cual es insuficiente para representar la sismicidad de una región, no obstante, el catálogo analizado en este estudio, es el más extenso existente en Costa Rica. Influye además en el valor $b$ la región geográfica seleccionada, ya que el valor obtenido es un promedio para todo el catálogo, y será distinto del valor que se calcule para regiones más pequeñas en las cuales lógicamente la cantidad y distribución de sismos serán distintas. En esta ocasión se ha dejado fuera de esta investigación la determinación de $b$ con base en una zonificación geográfica o por fuentes sísmicas.

Pese a las limitaciones aquí mencionadas, siendo la mayoría propias de los estudios que involucran el análisis de un catálogo sísmico, es relevante documentar el valor $b$ aproximado de un catálogo sísmico y poder así referenciar sus cambios conforme aparecen nuevas metodologías y las redes sismológicas capturan datos más completos. Durante los años 2016 y 2017, la RSN ha ampliado su red de estaciones con 90 nuevos instrumentos y esta porción del catálogo aún no ha sido analizada. La sismicidad registrada a partir de esta nueva ampliación permitirá aproximar más robustamente el valor $b$ en estudios futuros y corroborar los presentados en esta investigación con el uso de otras metodologías.

\section{CONCLUSIONES}

Con base en la metodología de Stepp (1972), se realizó un análisis de 111.850 sismos registrados por la RSN. Este catálogo fue depurado considerando varios factores como el área geográfica de interés y la calidad de las localizaciones. Además, el cálculo de la magnitud de los sismos fue homogeneizado a $M w$. El catálogo filtrado de acuerdo con las restricciones y los criterios mencionados en el artículo, está compuesto por 62.514 sismos.

Se determinó una $M C$ de 5,0 para el catálogo sísmico en el periodo 1975-2014. Además, se estableció que el catálogo es completo para el rango $4,5 \leq M w \leq 4,9$ en el periodo 1980-2014, para 3,5 $\leq M w \leq 4,4$ en el periodo 1990-2014, y para $3,0 \leq M w \leq 3,4$ en el periodo 19952014. El cambio de sistemas analógicos a digitales, a partir de 1990, y el aumento considerable en el número de estaciones, a partir del año 1995, está reflejado en los resultados obtenidos que muestran un valor menor de la $M C$ a partir de los años señalados.

El catálogo es incompleto para $M w \leq 2,5$, sin embargo, para $M w$ 2,5-2,9 durante el periodo 2000-2014 el catálogo podría estar completo, pero esto no se puede determinar con certeza por el método de Stepp (1972) porque el periodo de observación es muy corto. La inspección de la constancia en el tiempo de diversos rangos de $M w$ sugiere que los sismos de $M w$ de 4,8 y 4,9 están cerca de la completitud, y que en el caso particular de $M w$ 5,0-5,9 en el periodo 1975-1979, el catálogo podría ser incompleto.

A través de la metodología de Aki (1965), usando una $M C$ de 5,0 en la ventana de tiempo de 1975 al 2014, se determinó un valor $b$ de 0,96 , muy similar a los valores promedio regionales obtenidos en estudios previos $(0,97$ en Rojas et al., 1993; 0,91 en Laporte et al., 1994 y 0,93 en Benito et al., 2012). Algunos factores que influencian el sesgo del valor $b$ son: el uso de premonitores y réplicas de los terremotos, el cálculo de diversos tipos de magnitud y su uniformización, el tiempo de observación del catálogo y la región geográfica seleccionada. Es importante recalcar la importancia del entendimiento de estos factores en el cálculo de $M C$ y $b$, en miras de su correcta interpretación, especialmente para estudios de amenaza sísmica, pues tanto $M C$ como $b$, presentan variabilidad espacial y temporal debido a factores inherentes a la geotectónica de la región o a los que afectan la capacidad de detección de las redes instrumentales.

\section{AGRADECIMIENTOS}

Este estudio resulta, en primera instancia, de una iniciativa de investigación como parte de una Práctica Profesional Supervisada de la carrera en Ciencias Geográficas de la Universidad Nacional (UNA), por lo cual, agradecemos al personal docente de la Escuela de 
Ciencias Geográficas de la UNA (ECG) que nos apoyó en la realización del mismo. Este artículo es también el resultado de los proyectos de investigación Vigilancia Sísmica de Costa Rica (113-B5-704) y Estudio del catálogo de sismos de la Red Sismológica Nacional de Costa Rica (113-B5-A02) inscritos ante la Vicerrectoría de Investigación de la Universidad de Costa Rica. Agradecemos a todo el personal que ha colaborado con la RSN a lo largo de más de 30 años, tanto en la UCR como en el ICE y que han permitido el registro y localización de los sismos del catálogo analizado en este artículo.

\section{REFERENCIAS}

Aki, K. 1965. Maximum likelihood estimated of $b$ in the formula $\log \mathrm{N}=\mathrm{a}-\mathrm{bM}$ and its confidence limits. Bulletin of the Earthquake Research Institute, 43(2): 237-239.

Benito, B., y Jiménez, M. 1999. Peligrosidad sísmica. Física de la Tierra, 11: 13-47.

Benito, B., Lindholm, C., Camacho, E., Climent, A., Marroquin, G., Molina, E., Rojas, W., Escobar, J., Talavera, E., Alvarado, G., and Torres, Y. 2012. A new evaluation of seismic hazard for the Central America Region. Bulletin of the Seismological Society of America. 102(2): 504-523.

Caneva, A., Salcedo, E., Van Hissenhoven, R., y Alfaro, A. 2003. Estudio de sismicidad regional para el análisis de la amenaza sísmica para Bogotá. Ingeniería y Universidad, 7(2): 133-150.

Cárdenas, C., Garzón, Y., Santa, L., y Castillo, L. 2010. Modelo de Poisson para la ocurrencia y magnitud espacio-temporal de los sismos en Colombia. UD y la Geomática. 4: 28-43.

Chouliaras, G. 2009. Investigating the earthquake catalog of the National Observatory of Athens. Natural Hazards and Earth System Sciences, 9: 905-912.

Fan, G., Beck, S., and Wallace, T. 1993. The seismic source parameters of the 1991 Costa Rica aftershock sequence: Evidence for a transcurrent plate boundary. Journal of Geophysical Research, 98(B9): 15759-15778.

Franco, A., Lasserre, D., Lyon-Caen, H., Kostoglodov, V., Molina, E., Guzman-Speziale, M., Monterosso, D., Robles, V., Figueroa, C., Amaya, W., Barrier, E., Chiquin, L., Moran, S., Flores, O., Romero, J., Santiago, J.A., Manea, M., and Manea, V. C. 2012. Fault kinematics in northern Central America and coupling along the subduction interface of the Cocos Plate, from GPS data in Chiapas (Mexico), Guatemala and El Salvador. Geophysical Journal International, 189(3): 1223-1236.

Goes, S., Velasco, A., and Schwartz, S. 1993. The April 22, 1991, Valle de la Estrella, Costa Rica $\left(M_{w}\right.$ 7.7) earthquake and its tectonic implications: A broadband seismic study. Journal Geophysical Research, 98(B5): 8127-8142.

Gutenberg, B., and Richter, C. 1944. Frequency of earthquakes in California. Bulletin of the Seismological Society of America, 34(4): 185-188.

Knopoff, L. 2000. The magnitude distribution of declustered earthqaukes in Southern California. Proceedings of the National Academy Sciences USA, 97(22): 11880-11884.

Kulhanek, O. 2005. Seminar on b-value. Technical Report, Department of Geophysics, Charles University, Prague.

Laporte, M., Lindholm, C., Bungum, H., and Dahle, A. 1994 Seismic hazard for Costa Rica. NORSAR, Technical Report No. 2-14, 73p.

Lucke, O. 2014. Moho structure of Central America based on three-dimensional lithospheric density modelling of satellite-derived gravity data. International Journal of Earth Sciences, 103(7): 1733-1745.

Marshall, J., Fisher, D., and Gardner, T. 2000. Central Costa Rica deformed belt: Kinematics of diffuse faulting across the western Panama block. Tectonics, 19(3): 468-492.

Mendoza, A.R. 2012. Estudio de la estabilidad del valor $b$ para regiones sismotectónicas de México. Tesis de Maestría, Universidad Nacional Autónoma de México, México. 127p.

Mignan, A., and Woessner, J. 2012. Estimating the magnitude of completeness for earthquake catalogs. Community Online Resource for Statistical Seismicity Analysis, 4: 1-45.

Montero, W. 2001. Neotectónica de la región central de Costa Rica: Frontera oeste de la microplaca de Panamá. Revista Geológica de América Central, 24: 29-56.

Montero, W., y Rojas, W. 2014. Las fallas Purires y Picagres y su relación con la secuencia sísmica de Puriscal de 1990. Revista Geológica de América Central, 50: 39-69. 
Morales, L.D., 1986. Historia de la sismología en Costa Rica. Revista de Filosofía Universidad de Costa Rica, 24(59): 93-104.

Nasir, A., Lendhart, W., Hintersberger, E., and Decker, K. 2013. Assessing the completeness of historical and instrumental earthquake data in Austria and the surrounding areas. Austrian Journal of Earth Sciences, 106(1): 90-102.

Naylor, M., Orofanogiannaki, K., and Harte, D. 2010. Exploratory data analysis: Magnitude, space, and time. Community Online Resource for Statistical Seismicity Analysis, 3: 1-42.

Rojas, W. 1993. Catálogo de sismicidad histórica y reciente en América Central: Desarrollo y análisis. Tesis, Universidad de Costa Rica, Costa Rica. 91p.

Rojas, W., Cowan, H., Lindholm, C., Dahle, A., and Bungum, H. 1993. Regional seismic zonation for Central América: A preliminary model. NORSAR Report. Norway.

Sobiesiak, M. 2000. Fault plane structure of the Antofagasta, Chile Earthquake of 1995. Geophysical Research Letters, 27(4): 581-584.

Sobiesiak, M. 2005. Fault plane structure of the 1995 Antofagasta Earthquake (Chile) derived from local seismological parameters. Tesis Doctoral, Universitat Potsdam, Alemania. 137p.

Stepp, J.C. 1972. Analysis of completeness of the earthquake sample in the Puget Sound area and its effect on statistical estimates of earthquake hazard. Proceedings of the International Conference on Microzonation, 2: 897-910.

Valladares, R. 2013. Determinación de parámetros sísmicos asociados con la amenaza sísmica en la región nororiental de Venezuela. Tesis, Universidad de Oriente, Venezuela. $77 \mathrm{p}$.

Wiemer, S., and Wyss, M. 2000. Minimum magnitude of completeness in earthquake catalogs: Examples from Alaska, the Western United States, and Japan. Bulletin of the Seismological Society of America, 90(4): 859-869.

Wiemer, S. 2001. A software package to analyze seismicity: ZMAP. Seismological Research Letters, 72(3): 373-382.
Woessner, J., and Wiemer, S. 2005. Assessing the quality of earthquake catalogues: Estimating the magnitude of completeness and its uncertainty. Bulletin of the Seismological Society of America, 95(2): 684-698.

Trabajo recibido: abril 12 de 2017

Trabajo aceptado: junio 28 de 2017 